\title{
Inbreeding, Coancestry, and Founding Clones of Sweet Cherries from North America
}

\author{
Cheol Choi ${ }^{1}$ and Frank Kappel \\ Agriculture and Agri-Food Canada, Pacific Agri-Food Research Centre, Summerland, BC VOH 1Z0, Canada
}

AdDitional InDEX words. Prunus avium, breeding, genetic diversity, germplasm

Abstract. Inbreeding and coancestry coefficients were calculated for 66 sweet cherry (Prunus avium L.) selections released from four breeding programs in North America (HRIO, Vineland, Ont., IAREC, Prosser, Wash., NYSAES, Geneva, N.Y., and PARC, Summerland, B.C.). Highly used founding clones were 'Black Heart', 'Emperor Francis', 'Empress Eugenie', 'Napoleon' and 'Windsor'. Coefficients of coancestry between all selections and these clones averaged $\mathbf{0 . 0 3 8}, 0.045,0.060,0.091$, and 0.033 , respectively. In these five founding clones, coefficients of coancestry in self-compatible selections were over twice as much as those in self-incompatible selections except 'Windsor'. In the analysis of coefficients of coancestry between self-incompatible and self-compatible sweet cherry, almost $20 \%$ of self-incompatible selections represent more than a half-sib relationship $(0.125)$ to self-compatibles. Increasing and maintaining genetic diversity is needed in sweet cherry breeding program in North America for continued breeding progress.

In North America, cherry was introduced as seeds by early settlers in the northeastern United States and was later brought into the Midwest and Pacific West Coast (Hedrick, 1915). Sweet cherry (Prunus avium L.) improvement was initiated in North America by the Lewelling family in Oregon (Brown et al., 1996). 'Lambert' and 'Bing' were first selected from the seed of 'Napoleon' in about 1848 and 'Republican' in 1875, respectively (Hedrick, 1915). Early institutional sweet cherry breeding programs began at the New York State Agricultural Experiment Station, Geneva, in 1911; Horticultural Research Institute of Ontario, Vineland Station, Ont., Canada, in 1915; and Agriculture Agri-Food Canada, Summerland, B.C., in 1936 (Kappel and Lay, 1997; Wellington and Lamb, 1950).

Although most sweet cherries are self-incompatible and outbreeding diploids, inbreeding is increased when parents have common ancestors. The repeated use of a limited number of parents and their progeny as parents may decrease genetic diversity and increase inbreeding depression in future generations. Previous studies using molecular marker analysis showed a low degree of genetic diversity in sweet cherry cultivars (Gerlach and Stosser, 1998; Shimada et al., 1999; Zhou et al., 2002).

Although different regions have different breeding objectives, sweet cherry breeding in North America has recently mainly focused on self-compatibility, tolerance to rain-induced cracking, fruit size, fruit firmness, and disease resistance. The first selfcompatible sweet cherry seedlings were produced by a cross that was a result of 'Emperor Francis' seed donor and X-irradiation of 'Napoleon' pollen (Lewis and Crowe, 1954) at the John Innes Institute in the United Kingdom. After that, the first commercial self-compatible sweet cherry, 'Stella'('Lambert' X 'JI 2420' ), was introduced from the Pacific Agri-Food Research Centre (PARC), Summerland, B.C. (Lapins, 1970). All self-compatible selections released have been derived from 'Stella'. The data presented describes the level of inbreeding, coancestry and genetic contribution of founding clones among sweet cherry selections in

Received for publication 5 June 2003. Accepted for publication 3 Feb. 2004. Contribution number 2214 for AAFC-PARC. The authors thank Robert Andersen and Cheryl Hampson for their critical reading of the manuscript.

'Department of Horticulture, Hankyong National University, Ansung-city, Kyonggi-do, 456-749, Korea. To whom reprint requests should be addressed; e-mail choic@agr.gc.ca
Table 1. Parentage of 66 sweet cherry selections from four North American breeding programs.

\begin{tabular}{|c|c|c|}
\hline Selection & Parentage & $\begin{array}{c}\text { Breeding } \\
\text { programs }\end{array}$ \\
\hline $2 \mathrm{~N}-39-05$ & Van $x$ Stella & PARC \\
\hline $2 \mathrm{~N}-60-07$ & Bingy $x$ Stella & PARC \\
\hline $2 N-61-18$ & Star $\times$ Van & PARC \\
\hline $2 \mathrm{~N}-63-20$ & Bingy $x$ Salmo & PARC \\
\hline Benton & Stella $\times$ Beaulieu & IAREC \\
\hline Blackgold & Stark Gold x Stella & NYSAES \\
\hline Cashmere & Stella x Early Burlat & IAREC \\
\hline Celeste & Van $\times$ Newstar & PARC \\
\hline Chelan & Stella $\times$ Beaulieu & IAREC \\
\hline Chinook & Gil Peck x Bingy & IAREC \\
\hline Cristalina & Star $\times$ Van & PARC \\
\hline Gil Peck & Napoleon x Giant & NYSAES \\
\hline Glacier & Stella x Early Burlat & IAREC \\
\hline Hartland & Windsor $\mathrm{x}$ unknown & NYSAES \\
\hline Hudson & Oswego x Giant & NYSAES \\
\hline Index & Stella $\mathrm{X}$ unknown & IAREC \\
\hline Kristin & Emperor Francis $\mathrm{x}$ Gil Peck & NYSAES \\
\hline Lapins & Van $x$ Stella & PARC \\
\hline Newmoon & Van $\times$ Stella & PARC \\
\hline Newstar & Van $\times$ Stella & PARC \\
\hline NY1725 & Giant $x$ Emperor Francis & NYSAES \\
\hline Olympus & Lamberty $\mathbf{x}$ Van & IAREC \\
\hline P8-79 & Rainier $x$ Bingy & IAREC \\
\hline Rainier & Bingy $\mathbf{x}$ Van & IAREC \\
\hline Royalton & NY1725 $\mathrm{x}$ unknown & NYSAES \\
\hline Salmo & Lamberty $\mathbf{X}$ Van & PARC \\
\hline Sam & V1060140 x unknown & PARC \\
\hline Sandra Rose & 2N-61-18 x Sunburst & PARC \\
\hline Santina & Stella x Summit & PARC \\
\hline Selah & P8-79 x Stella & IAREC \\
\hline Simcoe & Stella $\mathrm{x}$ unknown & IAREC \\
\hline Skeena & $2 \mathrm{~N}-38-22$ y $x 2 \mathrm{~N}-60-07$ y & PARC \\
\hline Sodus & Napoleon x Giant & NYSAES \\
\hline Somerset & Van $x$ Vic & NYSAES \\
\hline Sonata & Lapins $x$ 2N-39-05 & PARC \\
\hline Sparkle & Empress Eugenie $\mathrm{x}$ unknown & PARC \\
\hline Staccato & Sweetheart $\mathrm{x}$ unknown & PARC \\
\hline Star & Deacon $\mathrm{x}$ unknown & PARC \\
\hline
\end{tabular}

continued next page 
Table 1 (continued). Parentage of 66 sweet cherry selections from four North American breeding programs.

\begin{tabular}{|c|c|c|}
\hline Selection & Parentage & $\begin{array}{l}\text { Breeding } \\
\text { programs }^{z}\end{array}$ \\
\hline Stardust & $2 \mathrm{~N}-63-20 \times$ Stella & PARC \\
\hline Stella & Lamberty $x$ JI2420y & PARC \\
\hline Sue & Bingy $x$ Schmidt & PARC \\
\hline Summer Jewel & $2 \mathrm{~N}-61-18^{\text {y }}$ x $2 \mathrm{~S}-28-30$ y & PARC \\
\hline Summit & Van $x$ Sam & PARC \\
\hline Sunburst & Van $\mathbf{x}$ Stella & PARC \\
\hline Sweetheart & Van $\times$ Newstar & PARC \\
\hline Sylvia & Van $x$ Sam & PARC \\
\hline Symphony & Lapins $x$ Bingy & PARC \\
\hline Tehranivee & Van $x$ Stella & HRIO \\
\hline Tieton & Stella $x$ Early Burlat & IAREC \\
\hline Ulster & Schmidt $x$ Lambert ${ }^{y}$ & NYSAES \\
\hline V1060140 & Windsor $\mathrm{x}$ unknown & HRIO \\
\hline V35024 & Hedelfingen $x$ Bing $^{y}$ & HRIO \\
\hline V35029 & Hedelfingen $x$ Bing $y$ & HRIO \\
\hline Valera & Hedelfingen $x$ Windsor & HRIO \\
\hline Van & Empress Eugenie $\mathrm{x}$ unknown & PARC \\
\hline Vandalay & Van $x$ Stella & HRIO \\
\hline Velvet & Windsor $\mathrm{x}$ unknown & HRIO \\
\hline Venus & Hedelfingen $\mathrm{x}$ Windsor & HRIO \\
\hline Vernon & Windsor $\mathrm{x}$ unknown & HRIO \\
\hline Vic & Bingy $\mathbf{X}$ Schmidt & HRIO \\
\hline Victor & Windsor $\mathrm{x}$ unknown & HRIO \\
\hline Viscount & V35024 x V35029 & HRIO \\
\hline Vista & Hedelfingen $\mathrm{x}$ Victor & HRIO \\
\hline Viva & Hedelfingen $\mathrm{x}$ Windsor & HRIO \\
\hline Vogue & Hedelfingen $\mathrm{x}$ Windsor & HRIO \\
\hline Whitegold & Emperor Francis $\mathrm{x}$ Stella & NYSAES \\
\hline
\end{tabular}

zIrrigated Agriculture Research and Extension Center (IAREC), Prosser, Wash.;Horticultural Research Institute of Ontario(HRIO), Vineland Station, Ont., Canada; New York State Agricultural Experiment Station (NYSAES), Geneva; Pacific Agri-Food Research Centre (PARC), Summerland, B.C., Canada.

y2N-38-22 = 'Van'x 'Stella';2N-60-07 = 'Bing' x 'Stella'; 2S-28-30 = 'Van' x 'Stella'; 'Bing' = 'Black Republican'x $x$ Unknown; J2420 = 'Emperor Francis' $x$ 'Napoleon' (mutated pollen); 'Lambert' = 'Napoleon' x 'Black Heart'.

×Black Republican was assumed to be the result of 'Napoleon' $\mathrm{x}$ 'Black Tartarian' (Hedrick, 1915).
North American breeding programs as previous studies in other fruit species (Byrne, 1989; Hancock and Siefker, 1982; Lansari et al., 1994; Noiton and Alspach, 1996; Scorza et al., 1985).

\section{Materials and Methods}

Pedigrees of 66 sweet cherry cultivars and breeding selections released from four breeding programs (15 from HRIO, Vineland, Ont., 12 from IAREC, Prosser, Wash., 11 from NYSAES, Geneva, N.Y., and 28 from PARC, Summerland, B.C.) in North America were collected from breeding records, published sources (Bargioni, 1996; Brooks and Olmo, 1972, 1997; Hedrick, 1915; Kappel and Lay, 1997), and via direct communication (R.L. Andersen and G. Lang, personal communication) (Table 1). 'Black Republican' was considered the result of 'Napoleon' by 'Black Tartarian' in this analysis (Hedrick, 1915). All parents of unknown origin were assumed to be noninbred and unrelated and also all open pollinations were assumed to be noninbred and unrelated to the pollen parent. These assumptions were based on the self-incompatibility in sweet cherry, may underestimate possible inbreeding and would give lower inbreeding coefficients. All 66 selections were classified into two groups by self-compatibility (42 self-incompatible and 24 self-compatible).

As with previous studies (Byrne, 1989; Scorza et al., 1985), the SAS procedure INBREED (Barr, 1983) was used to calculate inbreeding and coancestry coefficients. In this analysis, the inbreeding coefficient of an individual is the probability that a pair of alleles carried by the gametes that produced it are identical by descent (Falconer and Mackay, 1996) and is equal to the coancestry between its parents (Barr, 1983). The coancestry coefficient of prospective progeny of two individuals is equal to one half the covariance of the parents. Since the inbreeding coefficient of an individual is equal to the coancestry coefficient of its parents, the coancestry coefficient of two selections was calculated as the inbreeding coefficient of their prospective progeny (Lansari et al., 1994).

The inbreeding coefficients were calculated for all 66 sweet cherry selections, and the degree of relationship of these 66 selections with the five founding clones, 'Black Heart', 'Emperor Francis', 'Empress Eugenie', 'Napoleon', and 'Windor' was analyzed by calculation of coefficients of coancestry. Coefficients of coancesty

Table 2. Inbreeding coefficients and coancestry coefficients with 'Black Heart', 'Emperor Francis', 'Empress Eugenie', 'Napoleon' and 'Windsor' of 66 sweet cherry selections released from North American programs.

\begin{tabular}{|c|c|c|c|c|c|c|}
\hline \multirow[b]{3}{*}{ Selection } & \multicolumn{6}{|c|}{ Coefficient of coancestry ${ }^{y}$ with } \\
\hline & Inbreeding & Black & Emperor & Empress & & \\
\hline & coefficients & Heart & Francis & Eugenie & Napoleon & Windsor \\
\hline \multicolumn{7}{|c|}{ Self-incompatible } \\
\hline 2N-60-07 & 0.063 & 0.063 & 0.063 & --- & 0.188 & --- \\
\hline $2 \mathrm{~N}-61-18$ & $---z$ & --- & --- & 0.125 & --- & --- \\
\hline $2 \mathrm{~N}-63-20$ & 0.031 & 0.063 & --- & 0.063 & 0.125 & --- \\
\hline Chelan & --- & 0.063 & 0.063 & --- & 0.125 & --- \\
\hline Chinook & --- & --- & --- & --- & 0.188 & --- \\
\hline Cristalina & --- & --- & --- & 0.125 & --- & --- \\
\hline Gil Peck & --- & --- & --- & --- & 0.250 & --- \\
\hline Hartland & --- & --- & --- & --- & --- & 0.250 \\
\hline Hudson & --- & --- & --- & --- & --- & --- \\
\hline Kristin & --- & --- & 0.250 & --- & 0.125 & --- \\
\hline NY1725 & --- & --- & 0.250 & --- & --- & --- \\
\hline Olympus & --- & 0.125 & --- & 0.125 & 0.125 & --- \\
\hline P8-79 & 0.250 & --- & --- & 0.063 & 0.094 & --- \\
\hline Raininer & --- & --- & --- & 0.125 & 0.063 & --- \\
\hline Royalton & --- & --- & 0.125 & --- & --- & --- \\
\hline
\end{tabular}


Table 2 (continued). Inbreeding coefficients and coancestry coefficients with 'Black Heart', 'Emperor Francis', 'Empress Eugenie', 'Napoleon' and 'Windsor' of 66 sweet cherry selections released from North American programs.

\begin{tabular}{|c|c|c|c|c|c|c|}
\hline \multirow[b]{3}{*}{ Selection } & \multicolumn{6}{|c|}{ Coefficient of coancestry ${ }^{y}$ with } \\
\hline & Inbreeding & Black & Emperor & Empress & & \\
\hline & coefficients & Heart & Francis & Eugenie & Napoleon & Windsor \\
\hline Salmo & --- & 0.125 & --- & 0.125 & 0.125 & --- \\
\hline Sam & --- & --- & --- & --- & --- & 0.125 \\
\hline Simcoe & --- & 0.063 & 0.063 & --- & 0.125 & --- \\
\hline Sodus & --- & --- & --- & --- & 0.250 & --- \\
\hline Somerset & --- & --- & --- & 0.125 & 0.031 & --- \\
\hline Sparkle & --- & --- & --- & 0.250 & --- & --- \\
\hline Star & --- & --- & --- & --- & --- & --- \\
\hline Sue & --- & --- & --- & --- & 0.063 & --- \\
\hline Summer Jewel & 0.125 & 0.031 & 0.031 & 0.125 & 0.063 & --- \\
\hline Summit & --- & --- & --- & 0.125 & --- & 0.063 \\
\hline Sylvia & --- & --- & --- & 0.125 & --- & 0.063 \\
\hline Tieton & --- & 0.063 & 0.063 & --- & 0.125 & --- \\
\hline Ulster & --- & 0.125 & --- & --- & 0.125 & --- \\
\hline V1060140 & --- & --- & --- & --- & --- & 0.125 \\
\hline V35024 & --- & --- & --- & --- & 0.063 & --- \\
\hline V35029 & --- & --- & --- & --- & 0.063 & --- \\
\hline Valera & --- & --- & --- & --- & --- & 0.250 \\
\hline Van & --- & --- & --- & 0.250 & --- & --- \\
\hline Velvet & --- & --- & --- & --- & --- & 0.250 \\
\hline Venus & --- & --- & --- & --- & --- & 0.250 \\
\hline Vernon & --- & --- & --- & --- & --- & 0.250 \\
\hline Vic & --- & --- & --- & --- & 0.063 & --- \\
\hline Victor & --- & --- & --- & --- & --- & 0.250 \\
\hline Viscount & 0.250 & --- & --- & --- & 0.063 & --- \\
\hline Vista & --- & --- & --- & --- & --- & 0.125 \\
\hline Viva & --- & --- & --- & --- & --- & 0.250 \\
\hline Vogue & --- & --- & --- & --- & --- & 0.250 \\
\hline Mean & 0.144 & 0.017 & 0.022 & 0.042 & 0.058 & 0.063 \\
\hline \multicolumn{7}{|l|}{ Self-compatible } \\
\hline 2N-39-05 & --- & 0.063 & 0.063 & 0.125 & 0.125 & --- \\
\hline Benton & --- & 0.063 & 0.063 & --- & 0.125 & --- \\
\hline Blackgold & --- & 0.063 & 0.063 & --- & 0.125 & --- \\
\hline Cashmere & --- & 0.063 & 0.063 & --- & 0.125 & --- \\
\hline Celeste & 0.250 & 0.031 & 0.031 & 0.188 & 0.063 & --- \\
\hline Glacier & --- & 0.063 & 0.063 & --- & 0.125 & --- \\
\hline Index & --- & 0.063 & 0.063 & --- & 0.125 & --- \\
\hline Lapins & --- & 0.063 & 0.063 & 0.125 & 0.125 & --- \\
\hline Newmoon & --- & 0.063 & 0.063 & 0.125 & 0.125 & --- \\
\hline Newstar & --- & 0.063 & 0.063 & 0.125 & 0.125 & --- \\
\hline Sandra Rose & 0.125 & 0.031 & 0.031 & 0.125 & 0.063 & --- \\
\hline Santina & --- & 0.063 & 0.063 & 0.063 & 0.125 & 0.031 \\
\hline Selah & 0.047 & 0.063 & 0.063 & 0.031 & 0.172 & --- \\
\hline Skeena & 0.156 & 0.063 & 0.063 & 0.063 & 0.156 & --- \\
\hline Sonata & 0.266 & 0.063 & 0.063 & 0.125 & 0.125 & --- \\
\hline Staccato & --- & 0.016 & 0.016 & 0.094 & 0.031 & --- \\
\hline Stardust & 0.109 & 0.094 & 0.063 & 0.031 & 0.188 & --- \\
\hline Stella & 0.125 & 0.125 & 0.125 & --- & 0.250 & --- \\
\hline Sunburst & --- & 0.063 & 0.063 & 0.125 & 0.125 & --- \\
\hline Sweetheart & 0.250 & 0.031 & 0.031 & 0.188 & 0.063 & --- \\
\hline Symphony & 0.031 & 0.031 & 0.031 & 0.063 & 0.125 & --- \\
\hline Tehranivee & --- & 0.063 & 0.063 & 0.125 & 0.125 & --- \\
\hline Vandalay & --- & 0.063 & 0.063 & 0.125 & 0.125 & --- \\
\hline Whitegold & 0.125 & 0.063 & 0.313 & --- & 0.125 & --- \\
\hline Mean & 0.148 & 0.059 & 0.068 & 0.077 & 0.124 & 0.002 \\
\hline Grand Mean & 0.146 & 0.038 & 0.045 & 0.060 & 0.091 & 0.033 \\
\hline
\end{tabular}

${ }^{\mathrm{z}}$ Dashes indicate no inbreeding or coancestry of known parents.

yMean coefficients of coancestry calculated with dashes $=$ zero. 
Table 3. Coefficient of coancestry of self-incompatible sweet cherry selections released from North American breeding programs ${ }^{\mathrm{z}}$.

\begin{tabular}{|c|c|c|c|c|c|c|c|c|c|c|c|c|c|c|c|c|c|c|c|c|c|c|c|c|}
\hline Selection & $1 y$ & 2 & 3 & 4 & 5 & 6 & 7 & 8 & 9 & 10 & 11 & 12 & 13 & 14 & 15 & 16 & 17 & 18 & 19 & 20 & 21 & 22 & 23 & 24 \\
\hline 1) $2 \mathrm{~N}-60-07$ & 531 & ---w & 188 & 156 & 188 & --- & 94 & --- & --- & 78 & 31 & 94 & 211 & 141 & 16 & 94 & --- & 156 & 94 & 70 & --- & --- & 141 & 78 \\
\hline 2) $2 \mathrm{~N}-61-18$ & & 500 & 63 & --- & --- & 250 & --- & --- & --- & -.- & -- & 125 & 63 & 125 & --- & 125 & --- & --- & --- & 125 & 63 & 250 & --- & 313 \\
\hline 3) $2 \mathrm{~N}-63-20$ & & & 516 & 55 & 164 & 63 & 63 & --- & --- & 31 & --- & 141 & 230 & 195 & --- & 266 & --- & 55 & 63 & 129 & 31 & --- & 133 & 90 \\
\hline 4) Chelan & & & & 500 & 47 & --- & 63 & --- & --- & 63 & 31 & 78 & 23 & 16 & 16 & 78 & --- & 141 & 63 & 8 & --- & --- & 16 & 70 \\
\hline 5) Chinook & & & & & 531 & --- & 281 & --- & 63 & 141 & 63 & 47 & 211 & 141 & 31 & 47 & --- & 47 & 156 & 70 & --- & --- & 141 & 23 \\
\hline 6) Cristalina & & & & & & 500 & --- & --- & --- & --- & --- & 125 & 63 & 125 & --- & 125 & --- & --- & --- & 125 & 63 & 250 & --- & 188 \\
\hline 7) Gil Peck & & & & & & & 500 & --- & 125 & 250 & 125 & 63 & 47 & 31 & 63 & 63 & --- & 63 & 250 & 16 & --- & --- & 31 & 31 \\
\hline 8) Hartland & & & & & & & & 500 & --- & --- & --- & --- & --- & --- & --- & --- & 63 & --- & --- & --- & --- & --- & --- & -- \\
\hline 9) Hudson & & & & & & & & & 500 & 63 & 125 & --- & --- & --- & 63 & --- & --- & --- & 125 & --- & --- & --- & --- & --- \\
\hline 10) Kristin & & & & & & & & & & 500 & 188 & 31 & 23 & 16 & 94 & 31 & --- & 63 & 125 & 8 & --- & --- & 16 & 31 \\
\hline 11) NY1725 & & & & & & & & & & & 500 & --- & --- & --- & 250 & --- & --- & 31 & 125 & --- & --- & --- & --- & 16 \\
\hline 12) Olympus & & & & & & & & & & & & 500 & 86 & 141 & --- & 250 & --- & 78 & 63 & 133 & 63 & --- & 16 & 164 \\
\hline 13) P8-79 & & & & & & & & & & & & & 625 & 375 & --- & 86 & --- & 23 & 47 & 156 & 31 & --- & 188 & 74 \\
\hline 14) Rainier & & & & & & & & & & & & & & 500 & --- & 141 & --- & 16 & 31 & 188 & 63 & --- & 125 & 133 \\
\hline 15) Royalton & & & & & & & & & & & & & & & 500 & --- & --- & 16 & 63 & --- & --- & --- & --- & 8 \\
\hline 16) Salmo & & & & & & & & & & & & & & & & 500 & --- & 78 & 63 & 133 & 63 & --- & 16 & 164 \\
\hline 17) Sam & & & & & & & & & & & & & & & & & 500 & --- & --- & --- & --- & --- & -- & --- \\
\hline 18) Simcoe & & & & & & & & & & & & & & & & & & 500 & 63 & 8 & --- & --- & 16 & 70 \\
\hline 19) Sodus & & & & & & & & & & & & & & & & & & & 500 & 16 & --- & --- & 31 & 31 \\
\hline 20) Somerset & & & & & & & & & & & & & & & & & & & & 500 & 63 & --- & 125 & 129 \\
\hline 21) Sparkle & & & & & & & & & & & & & & & & & & & & & 500 & --- & --- & 63 \\
\hline 22) Star & & & & & & & & & & & & & & & & & & & & & & 500 & --- & 125 \\
\hline 23) Sue & & & & & & & & & & & & & & & & & & & & & & & 500 & 8 \\
\hline 24) Summer Jewel & & & & & & & & & & & & & & & & & & & & & & & & 563 \\
\hline \multicolumn{25}{|l|}{ 25) Summit } \\
\hline \multicolumn{25}{|l|}{ 26) Sylvia } \\
\hline \multicolumn{25}{|l|}{ 27) Tieton } \\
\hline \multicolumn{25}{|l|}{ 28) Ulster } \\
\hline \multicolumn{25}{|l|}{ 29) V1060140 } \\
\hline \multicolumn{25}{|l|}{ 30) V35024 } \\
\hline \multicolumn{25}{|l|}{ 31) V35029 } \\
\hline \multicolumn{25}{|l|}{ 32) Valera } \\
\hline \multicolumn{25}{|l|}{ 33) Van } \\
\hline \multicolumn{25}{|l|}{$\begin{array}{l}\text { 34) Velvet } \\
\text { 35) Venus }\end{array}$} \\
\hline \multicolumn{25}{|l|}{ 36) Vernon } \\
\hline \multicolumn{25}{|l|}{ 37) Vic } \\
\hline \multicolumn{25}{|l|}{ 38) Victor } \\
\hline \multicolumn{25}{|l|}{ 39) Viscount } \\
\hline 40) Vista & & & & & & & & & & & & & & & & & & & & & & & & \\
\hline 41) Viva & & & & & & & & & & & & & & & & & & & & & & & & \\
\hline 42) Vogue & & & & & & & & & & & & & & & & & & & & & & & & \\
\hline Mean & & & & & & & & & & & & & & & & & & & & & & & & \\
\hline
\end{tabular}

${ }^{2}$ Coefficients of coancestry values $\times 1000$.

y Numbers across top of table refer to selection numbers at far left of table.

xMean coefficient of coancestry excluding selfing.

wMean coefficient of coancestry calculated with dashes $=0$.

were also calculated among the 42 self-incompatibles, among 24 self-compatibles and among the 66 sweet cherries together.

\section{Results and Discussion}

Of the 66 selections analyzed, 15 ( 5 and 10 from self-incompatible and self-compatible selections, respectively) had an inbreeding coefficient other than zero (Table 2). The overall mean inbreeding coefficient was 0.146 for all selections and cultivars, where their parentages were known. If we assumed an inbreeding coefficient for selections of unknown parentage to be zero (nonrelated with known parentage), the overall mean inbreeding coefficients would be lowered (0.033). The mean for self-compatible and self-incompatible selections were 0.148 and 0.144 , respectively. These figures are high considering that a value of 0.063 would result from first cousin mating of noninbred, nonrelated diploids, 0.125 from half-sib mating, 0.250 from full-sibs, and 0.500 from selfing. Compared to other fruit species, the inbreeding level of sweet cherry is not as high as peach (0.26 to 0.35) (Scorza et al., 1985), but higher than blueberry (0.13) (Hancock and Siefker, 1982), raspberry (0.12) (Dale et al., 1993), plum (0.02 to 0.05) (Byrne, 1989), and apple (0.01 to 0.04) (Noiton and Alspach, 1996). It can be expected that the inbreeding level of future self-compatible selections will be elevated unless self-compatible genes other than S4' (mutation from 'Napoleon' pollen) are utilized in sweet cherry breeding programs.

Sixty-four selections (out of a total of 66) released from four sweet cherry breeding programs in North America were found to be descended from only five founding clones: 'Black Heart', 'Emperor Francis', 'Empress Eugenie', 'Napoleon', and 'Windsor'. These results suggest that these five founding clones have formed an exceptionally narrow germplasm base in the North American sweet cherry breeding program. Two cultivars, 'Hudson'(= 'Oswego' X 'Giant') and 'Star' (= 'Deacon' $x$ unknown) were not related to these five clones.

'Black Heart', one of the oldest cherries under cultivation, contributed to 9 of the 42 self-incompatible selections and $100 \%$ of the self-compatible selections. Along with 'Black Heart', 'Emperor Francis', and 'Napoleon' have also contributed to all of the self-compatible selections. The extensive use of 'Emperor Francis' 


\begin{tabular}{|c|c|c|c|c|c|c|c|c|c|c|c|c|c|c|c|c|c|c|}
\hline 25 & 26 & 27 & 28 & 29 & 30 & 31 & 32 & 33 & 34 & 35 & 36 & 37 & 38 & 39 & 40 & 41 & 42 & Meanx \\
\hline--- & --- & 156 & 94 & --- & 141 & 141 & --- & --- & --- & --- & --- & 141 & --- & 141 & --- & --- & --- & 67 \\
\hline 125 & 125 & --- & --- & --- & -- & --- & --- & 250 & --- & --- & --- & --- & --- & --- & --- & --- & --- & 48 \\
\hline 63 & 63 & 55 & 78 & --- & 133 & 133 & --- & 125 & --- & --- & --- & 133 & --- & 133 & --- & --- & --- & 70 \\
\hline --- & --- & 141 & 78 & --- & 16 & 16 & --- & --- & --- & --- & --- & 16 & --- & 16 & --- & --- & --- & 35 \\
\hline --- & --- & 47 & 47 & --- & 141 & 141 & --- & --- & --- & --- & --- & 141 & --- & 141 & --- & --- & --- & 61 \\
\hline 125 & 125 & --- & --- & --- & --- & --- & --- & 250 & --- & --- & --- & --- & --- & --- & --- & --- & --- & 45 \\
\hline --- & -- & 63 & 63 & --- & 31 & 31 & --- & --- & --- & --- & --- & 31 & --- & 31 & --- & --- & --- & 47 \\
\hline 31 & 31 & --- & --- & 125 & --- & --- & 125 & --- & 125 & 125 & 125 & --- & 125 & --- & 63 & 125 & 125 & 28 \\
\hline --- & --- & --- & --- & --- & --- & --- & --- & --- & --- & --- & -- & --- & --- & --- & --- & --- & -- & 13 \\
\hline --- & --- & 63 & 31 & --- & 16 & 16 & --- & --- & --- & --- & --- & 16 & --- & 16 & --- & --- & --- & 35 \\
\hline --- & --- & 31 & --- & --- & --- & --- & -- & --- & --- & --- & --- & --- & --- & --- & --- & --- & --- & 25 \\
\hline 125 & 125 & 78 & 125 & --- & 16 & 16 & --- & 250 & --- & --- & --- & 16 & --- & 16 & --- & --- & --- & 60 \\
\hline 63 & 63 & 23 & 23 & --- & 188 & 188 & --- & 125 & --- & --- & --- & 188 & --- & 188 & --- & --- & --- & 72 \\
\hline 125 & 125 & 16 & 16 & --- & 125 & 125 & --- & 250 & --- & --- & --- & 125 & --- & 125 & --- & --- & --- & 73 \\
\hline --- & --- & 16 & --- & --- & --- & --- & --- & --- & --- & --- & --- & --- & --- & --- & --- & --- & --- & 15 \\
\hline 125 & 125 & 78 & 125 & --- & 16 & 16 & --- & 250 & --- & --- & --- & 16 & --- & 16 & --- & --- & --- & 63 \\
\hline 250 & 250 & --- & --- & 250 & --- & --- & 63 & --- & 63 & 63 & 63 & --- & 63 & --- & 31 & 63 & 63 & 31 \\
\hline --- & --- & 141 & 78 & --- & 16 & 16 & --- & --- & --- & --- & --- & 16 & --- & 16 & --- & --- & --- & 32 \\
\hline --- & --- & 63 & 63 & --- & 31 & 31 & --- & --- & --- & --- & --- & 31 & --- & 31 & --- & --- & --- & 41 \\
\hline 125 & 125 & 8 & 70 & --- & 63 & 63 & --. & 250 & --- & --- & --- & 250 & --- & 63 & --- & --- & --- & 60 \\
\hline 63 & 63 & --- & --- & --- & --- & --- & --- & 125 & --- & --- & --- & --- & --- & --- & --- & --- & --- & 18 \\
\hline --- & --- & --- & --- & --- & --- & --- & --- & --- & --- & --- & --- & --- & --- & --- & --- & --- & --- & 15 \\
\hline --- & --- & 16 & 141 & --- & 125 & 125 & --- & --- & --- & --- & --- & 250 & --- & 125 & --- & --- & --- & 43 \\
\hline 125 & 125 & 70 & 39 & --- & 8 & 8 & --- & 250 & --- & --- & --. & 8 & --- & 8 & --- & --- & --- & 60 \\
\hline \multirow[t]{19}{*}{500} & 250 & --- & --- & 125 & --- & --- & 31 & 250 & 31 & 31 & 31 & --- & 31 & --- & 16 & 31 & 31 & 52 \\
\hline & 500 & --- & --- & 125 & --- & --- & 31 & 250 & 31 & 31 & 31 & --- & 31 & --- & 16 & 31 & 31 & 52 \\
\hline & & 500 & 78 & --- & 16 & 16 & --. & --- & -.- & --- & --. & 16 & --- & 16 & --- & --- & --. & 32 \\
\hline & & & 500 & --- & 16 & 16 & --- & --- & --- & --- & --- & 141 & --- & 16 & --- & --- & --- & 34 \\
\hline & & & & 500 & --- & --- & 125 & --- & 125 & 125 & 125 & --- & 125 & --- & 63 & 125 & 125 & 37 \\
\hline & & & & & 500 & 250 & 125 & --- & --- & 125 & --. & 125 & --- & 375 & 125 & 125 & 125 & 59 \\
\hline & & & & & & 500 & 125 & --- & --- & 125 & --- & 125 & --- & 375 & 125 & 125 & 125 & 59 \\
\hline & & & & & & & 500 & --- & 125 & 250 & 125 & --- & 125 & 125 & 188 & 250 & 250 & 49 \\
\hline & & & & & & & & 500 & --- & --- & --- & --- & --- & --- & --- & --- & --- & 63 \\
\hline & & & & & & & & & 500 & 125 & 125 & --- & 125 & --- & 63 & 125 & 125 & 28 \\
\hline & & & & & & & & & & 500 & 125 & --- & 125 & 125 & 188 & 250 & 250 & 49 \\
\hline & & & & & & & & & & & 500 & --- & 125 & --- & 63 & 125 & 125 & 28 \\
\hline & & & & & & & & & & & & 500 & --- & 125 & --- & --- & --- & 46 \\
\hline & & & & & & & & & & & & & 500 & --- & 250 & 125 & 125 & 33 \\
\hline & & & & & & & & & & & & & & 625 & 125 & 125 & 125 & 62 \\
\hline & & & & & & & & & & & & & & & 500 & 188 & 188 & 40 \\
\hline & & & & & & & & & & & & & & & & 500 & 250 & 49 \\
\hline & & & & & & & & & & & & & & & & & 500 & 49 \\
\hline & & & & & & & & & & & & & & & & & & 45 \\
\hline
\end{tabular}

and 'Napoleon' can be explained by the original creation of selfcompatibility in sweet cherry. 'Emperor Francis' contributed to 8 of the 42 self-incompatible selections. 'Napoleon' also known as 'Royal Ann', grown in Europe in the early 18th century, was most frequently found in the pedigree of self-incompatible selections in North America (21 of 42). Although 'Napoleon' has less desirable traits such as susceptibility to brown rot and cracking, it has outstanding breeding material characteristics, including firm flesh, large size, and attractive appearance of the fruit, along with good productivity of the trees (Hedrick, 1915). This cultivar has been grown largely for processing (Bargioni, 1996). 'Empress Eugenie' was found in the development of many cultivars from PARC, Summerland, B.C. However, none of the cultivars directly used 'Empress Eugenie' as a parent except 'Sparkle' and 'Van'. Due to its good fruit characteristics (i.e., fruit size, firmness, and flavor) and tree hardiness (Lapins et al., 1977), 'Van' ('Empress Eugenie' $x$ unknown) was used extensively in North American breeding programs and was an intermediate parent in $80 \%$ of the cultivars and selections developed at Summerland, B.C. 'Windsor' was almost exclusively used in the cherry breeding program at HRIO (9 of 15), the region where the cultivar originated (Hedrick, 1915).

The average coancestry within self-incompatible sweet cherry is 0.045 and mean coefficients range from 0.013 to 0.073 (Table 3 ). These values were comparable with coancestry of apples in the 50 world main-stream cultivars (average 0.052 ; range 0.006 to 0.090 ) and $V f$-carrier (average 0.051 ; range 0.017 to 0.088 ) groups (Noiton and Alspach, 1996). Coefficients of coancestry among self-compatible selections range from 0.102 to 0.256 (Table 4), and these values were much higher than coancestry in self-incompatible sweet cherry selections (0.013 to 0.073 ) or plums (0.069 to 0.080) (Byrne, 1989) and comparable with average coancestry reported for peaches $(0.023$ to $0.208,0.034$ to 0.330) (Scorza et al., 1985). In the case of peaches, some cultivars were reported or assumed to be the result of self-pollinations; however, there are no reports that sweet cherry selections were the result of self-pollination. Without any self-pollination, the average coancestry within self-compatible selections is 0.179 and it is considered quite high ( $0.125=$ half-sib relationship). Only one out of 22 self-compatible selections had coefficients of 
Table 4. Coefficient of coancestry of self-compatible sweet cherry selections released from North American breeding programs ${ }^{\mathrm{z}}$.

\begin{tabular}{|c|c|c|c|c|c|c|c|c|c|c|c|c|}
\hline Selection & $1 \mathrm{y}$ & 2 & 3 & 4 & 5 & 6 & 7 & 8 & 9 & 10 & 11 & 12 \\
\hline 1) $2 \mathrm{~N}-39-05$ & 500 & 141 & 141 & 141 & 258 & 141 & 141 & 266 & 266 & 266 & 195 & 203 \\
\hline 2) Benton & & 500 & 141 & 141 & 70 & 141 & 141 & 141 & 141 & 141 & 70 & 14 \\
\hline 3) Blackgold & & & 500 & 141 & 70 & 141 & 141 & 141 & 141 & 141 & 70 & \\
\hline 4) Cashmere & & & & 500 & 70 & 266 & 141 & 141 & 141 & 141 & 70 & 14 \\
\hline 5) Celeste & & & & & 625 & 70 & 70 & 258 & 258 & 375 & 223 & \\
\hline 6) Glacier & & & & & & 500 & 141 & 141 & 141 & 141 & 70 & 12 \\
\hline 7) Index & & & & & & 500 & 141 & 141 & 141 & 70 & 141 & \\
\hline 8) Lapins & & & & & & & & 500 & 266 & 266 & 195 & \\
\hline 9) Newmoon & & & & & & & & & 500 & 266 & 195 & 203 \\
\hline 10) Newstar & & & & & & & & & & 500 & 195 & 2 \\
\hline 11) Sandra Rose & & & & & & & & & & & 563 & 133 \\
\hline 12) Santina & & & & & & & & & & & & 500 \\
\hline 13) Selah & & & & & & & & & & & & \\
\hline 14) Skeena & & & & & & & & & & & & \\
\hline 15) Sonata & & & & & & & & & & & & \\
\hline 16) Staccato & & & & & & & & & & & & \\
\hline 17) Stardust & & & & & & & & & & & & \\
\hline 18) Stella & & & & & & & & & & & & \\
\hline 19) Sunburst & & & & & & & & & & & & \\
\hline 20) Sweetheart & & & & & & & & & & & & \\
\hline 21) Symphony & & & & & & & & & & & & \\
\hline 22) Tehranivee & & & & & & & & & & & & \\
\hline 23) Vandalay & & & & & & & & & & & & \\
\hline 24) Whitegold & & & & & & & & & & & & \\
\hline Mean coefficent $\mathrm{c}$ & & & & & & & & & & & & \\
\hline
\end{tabular}

zoefficients of coancestry values X 1000 .

yNumbers across top of table refer to genotype numbers at far left of table.

xMean coefficient of coancestry excluding selfing.

coancestry less than a half-sib relationship (0.125). 'Stella', the first commercial self-compatible cultivar, recorded the highest coefficient of coancestry value $(0.256)$, which is greater than a parent-offspring relationship (0.250).

The coefficients of coancestry between self-incompatible and self-compatible sweet cherry range from 0 to 0.168 with an overall mean of 0.060 (Table 5). In the self-compatible selections, all selections were quite consistently related to self-incompatible selections (0.035 to 0.077). Eight out of 42 self-incompatible selections were more closely related to self-compatible groups (i.e., coancestry $>0.125$; half-sib relationship). These numbers may be underestimated because we assumed unknown or open pollinated parents were not related with the known parent.

The inbreeding problem and potential genetic limitations have been raised for numerous other fruit species (Lansari et al., 1994; Noiton et al., 1994). Our results suggest the repeated use of five founding clones and one genetic source for self-compatibility has narrowed the genetic base of sweet cheery breeding in North America. As the gene pool gets narrower and narrower so does the genetic gain. A lack of diverse germplasm may limit long-term progress in sweet cherry breeding programs.

In sweet cherry breeding programs, redundant use of the same parents and their progeny can greatly limit prospects for continued success (Andersen, 1998). For continued improvement of commercial traits in sweet cherry genetic diversity should be maximized and loss of diversity minimized. Possible methods to accomplish this include using more diverse parents in crossing, using the other self-compatible sweet cherry allele(s) that the John Innes Institute developed (Tehrani and Brown, 1992), and searching for naturally occurring self-compatible mutants in sweet cherry such as in sour cherry (Yamane et al., 2003) and Japanese apricot (Tao et al., 2002). Maximizing genetic diversity is important, since it enhances the potential gain from selection (Lansari et al., 1994). Unexposed (wild) germplasm should be collected and introduced in future sweet cherry breeding programs. To minimize the loss of genetic diversity, one could adopt a modified backcross design to avoid inbreeding (Crosby et al., 1992) or a recurrent selection for combining ability as in an apple breeding population (Noiton and Shelbourne, 1992).

\section{Literature Cited}

Andersen, R.L. 1998. Current status of sweet cherry breeding. Acta Hort. 468:35-44.

Bargioni, G. 1996. Sweet cherry scions: Characteristics of the principal commercial cultivars, breeding objectives and methods, p. 73-112. In: A.D. Webster and N.E. Looney (eds.). Cherries: Crop physiology, production and uses. CAB Intl., Wallingford, U.K.

Barr, A.J. 1983. The INBREED procedure, p. 121-130. In: S.P. Joyner (ed.). SUGI supplemental library user's guide. SAS Inst., Cary, N.C.

Brooks, R.M. and H.P. Olmo. 1972. Register of new fruit and nut varieties. $2^{\text {nd }}$ ed. Univ. Calif. Press, Berkeley.

Brooks, R.M. and H.P. Olmo. 1997. Register of new fruit and nut varieties, $3^{\text {rd }}$ ed. ASHS Press, Alexandria, Va.

Brown, S.K., A.F. Iezzoni, and H.W. Fogle. 1996. Cherries, p. 213-255. In: J. Janick and J.M. Moore (eds.). Fruit breeding. vol. 1. Tree and tropical fruits. Wiley, New York.

Byrne, D.H. 1989. Inbreeding, coancestry, and founding clones of Japanese-type plums of California and the southeastern United States. J. Amer. Soc. Hort. Sci. 114:699-705. 


\begin{tabular}{|c|c|c|c|c|c|c|c|c|c|c|c|c|}
\hline 13 & 14 & 15 & 16 & 17 & 18 & 19 & 20 & 21 & 22 & 23 & 24 & Meanx \\
\hline 184 & 211 & 383 & 129 & 199 & 281 & 266 & 258 & 148 & 266 & 266 & 172 & 214 \\
\hline 152 & 148 & 141 & 35 & 168 & 281 & 141 & 70 & 86 & 141 & 141 & 172 & 134 \\
\hline 152 & 148 & 141 & 35 & 168 & 281 & 141 & 70 & 86 & 141 & 141 & 172 & 134 \\
\hline 152 & 148 & 141 & 35 & 168 & 281 & 141 & 70 & 86 & 141 & 141 & 172 & 139 \\
\hline 123 & 168 & 258 & 188 & 131 & 141 & 258 & 375 & 137 & 258 & 258 & 86 & 185 \\
\hline 152 & 148 & 141 & 35 & 168 & 281 & 141 & 70 & 86 & 141 & 141 & 172 & 139 \\
\hline 148 & 141 & 35 & 168 & 281 & 141 & 70 & 86 & 141 & 141 & 172 & 134 & \\
\hline 184 & 211 & 383 & 129 & 199 & 281 & 266 & 258 & 266 & 266 & 266 & 172 & 219 \\
\hline 184 & 211 & 266 & 129 & 199 & 281 & 266 & 258 & 148 & 266 & 266 & 172 & 209 \\
\hline 184 & 211 & 266 & 188 & 199 & 281 & 266 & 375 & 148 & 266 & 266 & 172 & 221 \\
\hline 107 & 137 & 195 & 111 & 115 & 141 & 313 & 223 & 105 & 195 & 195 & 86 & 148 \\
\hline 168 & 180 & 203 & 82 & 184 & 281 & 203 & 164 & 117 & 203 & 203 & 172 & 173 \\
\hline \multirow[t]{13}{*}{523} & 223 & 184 & 62 & 237 & 305 & 184 & 123 & 201 & 184 & 184 & 184 & 172 \\
\hline & 578 & 211 & 84 & 225 & 297 & 211 & 168 & 184 & 211 & 211 & 180 & 186 \\
\hline & & 633 & 129 & 199 & 281 & 266 & 258 & 207 & 266 & 266 & 172 & 221 \\
\hline & & & 500 & 65 & 70 & 129 & 313 & 68 & 129 & 129 & 43 & 102 \\
\hline & & & & 555 & 336 & 199 & 131 & 182 & 199 & 199 & 199 & 184 \\
\hline & & & & & 563 & 281 & 141 & 172 & 281 & 281 & 344 & 256 \\
\hline & & & & & & 500 & 258 & 148 & 266 & 266 & 172 & 214 \\
\hline & & & & & & & 625 & 137 & 258 & 258 & 86 & 191 \\
\hline & & & & & & & & 516 & 148 & 148 & 102 & 139 \\
\hline & & & & & & & & & 500 & 266 & 172 & 209 \\
\hline & & & & & & & & & & 500 & 172 & 209 \\
\hline & & & & & & & & & & & 563 & 162 \\
\hline & & & & & & & & & & & & 179 \\
\hline
\end{tabular}

Crosby, J.A., J. Janick, P.C. Pecknold, S.S. Korban, P.A. O'Connor, S.M. Ries, J. Goffreda, and A. Voordeckers. 1992. Breeding apples for scab resistance: 1945-1990. Fruit Var. J. 46:145-166.

Dale, A., P.P. Moore, R.J. McNicol, T.M. Sjulin, and L.A. Burmistrov. 1993. Genetic diversity of red raspberry varieties throughout the world. J. Amer. Soc. Hort. Sci. 118:119-129.

Falconer, D.S. and T.F.C. Mackay. 1996. Introduction to quantitative genetics, $4^{\text {th }}$ ed. Longman, New York.

Gerlach, H.K. and R. Stosser. 1998. Sweet cherry cultivar identification using RAPD-derived DNA fingerprints. Acta Hort. 468:63-69.

Hancock, J.F. and J.H. Siefker. 1982. Levels of inbreeding in highbush blueberry cultivars. HortScience 17:363-366.

Hedrick, U.P. 1915. The cherries of New York. N.Y. Agr. Expt. Sta., Geneva.

Kappel, F. and W. Lay. 1997. Sweet cherry breeding in Canada from the early 1900 s to 1994 . Fruit Var. J. 51:233-238.

Lansari, A., D.E. Kester, and A.F. Iezzoni. 1994. Inbreeding, coancestry, and founding clones of almonds of California, Mediterranean Shores, and Russia. J. Amer. Soc. Hort. Sci. 119:1279-1285.

Lapins, K.O. 1970. The 'Stella' cherry. Fruit Var. Hort. Dig. 24:19-20.

Lapins, K.O., L.G. Denby, and W.D. Lane. 1977. Fruit tree cultivars in British Columbia. Agr. Can. Publ. 1609. Can. Dept. Agr., Ottawa.

Lewis, D. and L.K. Crowe. 1954. The induction of self-fertility in tree fruits. J. Hort. Sci. 29:220-225.

Noiton, D.A.M. and C.G.A. Shelbourne. 1992. Quantitative genetics in an apple breeding strategy. Euphytica 60:213-219.

Noiton, D.A.M. and P.A. Alspach. 1996. Founding clones, inbreeding, coancestry, and status number of modern apple cultuvars. J. Amer. Soc. Hort. Sci. 121:773-782.

Noiton, D.A.M., A. Currie, and T. Shelbourne. 1994. Genetic diversity for apple breeding. Orchardist of New Zealand 67:36-39.

Scorza, R., W.B. Sherman, and G.W. Lightner. 1985. Inbreeding and coancestry of freestone peach cultivars of the eastern United States and implications for peach germplasm improvement. J. Amer. Soc. Hort. Sci. 110:547-552.

Shimada T., T. Shiratori, H. Hayama, K. Nishimura, M. Yamaguchi, and M. Yoshida. 1999. Genetic diversity of cherries characterized by random amplified polymorphic DNA (RAPD) analysis. J. Jpn. Soc. Hort. Sci. 68:984-986.

Tao, R., A. Namba, T. Habu, H. Yamane, and A. Sugiura. 2002. Sf-RNase and its relation to self-compatibility in Japanese apricot, p. 339. In: Abstr. XXVI ${ }^{\text {th }}$ Intl. Hort. Congr., Toronto, Canada. 11-17 Aug. 2002.

Tehrani, G. and S.K. Brown. 1992. Pollen-incompatibility and self-fertility in sweet cherry. Plant Breeding Rev. 9:367-388

Wellington, R. and R.C. Lamb. 1950. Sweet cherry breeding. Proc. Amer. Soc. Hort. Sci. 55:263-264.

Yamane, H., R.K. Ikeda, N.R. Hauck, A.F. Iezzoni, and R. Tao. 2003. Self-incompatibility $(S)$ locus region of the mutated $\mathrm{S}^{6}$-haplotype of sour cherry (Prunus cerasus) contains a functional pollen $S$ allele and a nonfunctional pistil $S$ allele. J. Expt. Bot. 54:2431-2437.

Zhou, L., F. Kappel, C. Hampson, P.A. Wiersma, and G. Bakkeren. 2002. Genetic analysis and discrimination of sweet cherry cultivars and selections using amplified fragment length polymorphism fingerprints. J. Amer. Soc. Hort. 127:786-792. 
Table 5. Coefficient of coancestry of 66 selected sweet cherry selections released from North American breeding programs.

\begin{tabular}{|c|c|c|c|c|c|c|c|c|c|c|c|c|c|}
\hline Selection & $2 \mathrm{~N}-39-05$ & Benton & Blackgold & Cashmere & Celeste & Glacier & Index & Lapins & Newmoon & Newstar & Sandra Rose & Santina & Selah \\
\hline $2 \mathrm{~N}-60-07$ & 156 & 156 & 156 & 156 & 78 & 156 & 156 & 156 & 156 & 156 & 78 & 156 & 262 \\
\hline $2 \mathrm{~N}-61-18$ & 125 & $---y$ & --- & --- & 188 & --- & --- & 125 & 125 & 125 & 313 & 63 & 31 \\
\hline $2 \mathrm{~N}-63-20$ & 117 & 55 & 55 & 55 & 121 & 55 & 55 & 117 & 117 & 117 & 90 & 86 & 170 \\
\hline Chelan & 141 & 266 & 141 & 141 & 70 & 141 & 141 & 141 & 141 & 141 & 70 & 141 & 152 \\
\hline Chinook & 47 & 47 & 47 & 47 & 23 & 47 & 47 & 47 & 47 & 47 & 23 & 47 & 152 \\
\hline Cristalina & 125 & --- & --- & --- & 188 & --- & --- & 125 & 125 & 125 & 188 & 63 & 31 \\
\hline Gil Peck & 63 & 63 & 63 & 63 & 31 & 63 & 63 & 63 & 63 & 63 & 31 & 63 & 86 \\
\hline Hartland & --- & --- & --- & --- & --- & --- & --- & --- & --- & --- & --- & 16 & --- \\
\hline Hudson & --- & --- & --- & --- & --- & --- & --- & --- & --- & --- & --- & --- & --- \\
\hline Kristin & 63 & 63 & 63 & 63 & 31 & 63 & 63 & 63 & 63 & 63 & 31 & 63 & 74 \\
\hline NY1725 & 31 & 31 & 31 & 31 & 16 & 31 & 31 & 31 & 31 & 31 & 16 & 31 & 31 \\
\hline Olympus & 203 & 78 & 78 & 78 & 227 & 78 & 78 & 203 & 203 & 203 & 164 & 141 & 121 \\
\hline P8-79 & 86 & 23 & 23 & 23 & 105 & 23 & 23 & 86 & 86 & 86 & 74 & 55 & 336 \\
\hline Rainier & 141 & 16 & 16 & 16 & 195 & 16 & 16 & 141 & 141 & 141 & 133 & 78 & 203 \\
\hline Royalton & 16 & 16 & 16 & 16 & 8 & 16 & 16 & 16 & 16 & 16 & 8 & 16 & 16 \\
\hline Salmo & 203 & 78 & 78 & 78 & 227 & 78 & 78 & 203 & 203 & 203 & 164 & 141 & 121 \\
\hline Sam & --- & --- & --- & --- & --- & --- & --- & --- & --- & --- & --- & 125 & --- \\
\hline Simcoe & 141 & 141 & 141 & 141 & 70 & 141 & 141 & 141 & 141 & 141 & 70 & 141 & 152 \\
\hline Sodus & 63 & 63 & 63 & 63 & 31 & 63 & 63 & 63 & 63 & 63 & 31 & 63 & 86 \\
\hline Somerset & 133 & 8 & 8 & 8 & 191 & 8 & 8 & 133 & 133 & 133 & 129 & 70 & 86 \\
\hline Sparkle & 63 & --- & --- & --- & 94 & --- & --- & 63 & 63 & 63 & 63 & 31 & 16 \\
\hline Star & --- & --- & --- & --- & --- & --- & --- & --- & --- & --- & 125 & --- & --- \\
\hline Sue & 16 & 16 & 16 & 16 & 8 & 16 & 16 & 16 & 16 & 16 & 8 & 16 & 109 \\
\hline Summer Jewel & 195 & 70 & 70 & 70 & 223 & 70 & 70 & 195 & 195 & 195 & 254 & 133 & 107 \\
\hline Summit & 125 & --- & --- & --- & 188 & --- & --- & 125 & 125 & 125 & 125 & 250 & 31 \\
\hline Sylvia & 125 & --- & --- & --- & 188 & --- & --- & 125 & 125 & 125 & 125 & 125 & 31 \\
\hline Tieton & 141 & 141 & 141 & 266 & 70 & 266 & 141 & 141 & 141 & 141 & 70 & 141 & 152 \\
\hline Ulster & 78 & 78 & 78 & 78 & 39 & 78 & 78 & 78 & 78 & 78 & 39 & 78 & 90 \\
\hline V1060140 & --- & --- & --- & --- & --- & --- & --- & --- & --- & --- & --- & 63 & --- \\
\hline V35024 & 16 & 16 & 16 & 16 & 8 & 16 & 16 & 16 & 16 & 16 & 8 & 16 & 109 \\
\hline V35029 & 16 & 16 & 16 & 16 & 8 & 16 & 16 & 16 & 16 & 16 & 8 & 16 & 109 \\
\hline Valera & --- & --- & --- & --- & --- & --- & --- & --- & --- & --- & --- & 16 & --- \\
\hline Van & 250 & --- & --- & --- & 375 & --- & --- & 250 & 250 & 250 & 250 & 125 & 63 \\
\hline Velvet & --- & --- & --- & --- & --- & --- & --- & --- & --- & --- & --- & 16 & --- \\
\hline Venus & --- & --- & --- & --- & --- & --- & --- & --- & --- & --- & --- & 16 & --- \\
\hline Vernon & --- & --- & --- & --- & --- & --- & --- & --- & --- & --- & --- & 16 & --- \\
\hline Vic & 16 & 16 & 16 & 16 & 8 & 16 & 16 & 16 & 16 & 16 & 8 & 16 & 109 \\
\hline Victor & --- & --- & --- & --- & --- & --- & --- & --- & --- & --- & --- & 16 & --- \\
\hline Viscount & 16 & 16 & 16 & 16 & 8 & 16 & 16 & 16 & 16 & 16 & 8 & 16 & 109 \\
\hline Vista & --- & --- & --- & --- & --- & --- & --- & --- & --- & --- & --- & 8 & --- \\
\hline Viva & --- & --- & --- & --- & --- & --- & --- & --- & --- & --- & --- & 16 & --- \\
\hline Vogue & --- & --- & --- & --- & --- & --- & --- & --- & --- & --- & --- & 16 & --- \\
\hline Mean & 71 & 46 & 35 & 37 & 72 & 37 & 35 & 71 & 71 & 71 & 64 & 66 & 77 \\
\hline
\end{tabular}

${ }^{2}$ Coefficients of coancestry values X 1000 .

yMean coefficient of coancestry calculated with dashes $=0$. 


\begin{tabular}{|c|c|c|c|c|c|c|c|c|c|c|c|}
\hline Skeena & Sonata & Staccato & Stardust & Stella & Sunburst & Sweetheart & Symphony & Tehranivee & Vandalay & Whitegold & Mean \\
\hline 344 & 156 & 39 & 250 & 313 & 156 & 78 & 219 & 156 & 156 & 188 & 168 \\
\hline 63 & 125 & 94 & 31 & --- & 125 & 188 & 63 & 125 & 125 & --- & 85 \\
\hline 152 & 117 & 61 & 313 & 109 & 117 & 121 & 191 & 117 & 117 & 55 & 112 \\
\hline 117 & 47 & 12 & 129 & 94 & 47 & 23 & 164 & 47 & 47 & 47 & 60 \\
\hline 63 & 125 & 94 & 31 & --- & 125 & 188 & 63 & 125 & 125 & --- & 79 \\
\hline 78 & 63 & 16 & 94 & 125 & 63 & 31 & 63 & 63 & 63 & 63 & 62 \\
\hline --- & --- & --- & --- & --- & --- & --- & --- & --- & --- & --- & 1 \\
\hline --- & --- & --- & --- & --- & --- & --- & --- & --- & --- & --- & 0 \\
\hline 70 & 63 & 16 & 78 & 125 & 63 & 31 & 47 & 63 & 63 & 188 & 65 \\
\hline 31 & 31 & 8 & 31 & 63 & 31 & 16 & 16 & 31 & 31 & 156 & 34 \\
\hline 148 & 203 & 113 & 148 & 156 & 203 & 227 & 117 & 203 & 203 & 78 & 152 \\
\hline 148 & 86 & 53 & 139 & 47 & 86 & 105 & 230 & 86 & 86 & 23 & 88 \\
\hline 141 & 141 & 98 & 113 & 31 & 141 & 195 & 195 & 141 & 141 & 16 & 108 \\
\hline 16 & 16 & 4 & 16 & 31 & 16 & 8 & 8 & 16 & 16 & 78 & 17 \\
\hline 148 & 203 & 113 & 211 & 156 & 203 & 227 & 117 & 203 & 203 & 78 & 155 \\
\hline 148 & 141 & 35 & 168 & 281 & 141 & 70 & 86 & 141 & 141 & 172 & 134 \\
\hline 78 & 63 & 16 & 94 & 125 & 63 & 31 & 63 & 63 & 63 & 63 & 62 \\
\hline 102 & 133 & 96 & 72 & 16 & 133 & 191 & 129 & 133 & 133 & 8 & 91 \\
\hline 31 & 63 & 47 & 16 & --- & 63 & 94 & 31 & 63 & 63 & --- & 38 \\
\hline --- & --- & --- & --- & --- & --- & --- & --- & --- & --- & --- & 5 \\
\hline 78 & 16 & 4 & 82 & 31 & 16 & 8 & 133 & 16 & 16 & 16 & 29 \\
\hline 137 & 195 & 111 & 115 & 141 & 195 & 223 & 105 & 195 & 195 & 86 & 148 \\
\hline 63 & 125 & 94 & 31 & --- & 125 & 188 & 63 & 125 & 125 & --- & 85 \\
\hline 63 & 125 & 94 & 31 & --- & 125 & 188 & 63 & 125 & 125 & --- & 79 \\
\hline 148 & 141 & 35 & 168 & 281 & 141 & 70 & 86 & 141 & 141 & 172 & 145 \\
\hline 86 & 78 & 20 & 117 & 156 & 78 & 39 & 55 & 78 & 78 & 78 & 76 \\
\hline --- & --- & --- & --- & --- & --- & --- & --- & --- & --- & --- & 3 \\
\hline 78 & 16 & 4 & 82 & 31 & 16 & 8 & 133 & 16 & 16 & 16 & 29 \\
\hline 78 & 16 & 4 & 82 & 31 & 16 & 8 & 133 & 16 & 16 & 16 & 29 \\
\hline --- & --- & --- & --- & --- & --- & --- & --- & --- & --- & --- & 1 \\
\hline 125 & 250 & 188 & 63 & --- & 250 & 375 & 125 & 250 & 250 & --- & 154 \\
\hline 78 & 16 & 4 & 82 & 31 & 16 & 8 & 133 & 16 & 16 & 16 & 29 \\
\hline --- & --- & --- & --- & --- & --- & --- & --- & --- & --- & --- & 1 \\
\hline 78 & 16 & 4 & 82 & 31 & 16 & 8 & 133 & 16 & 16 & 16 & 29 \\
\hline --- & --- & --- & --- & --- & --- & --- & --- & --- & --- & --- & 0 \\
\hline --- & --- & --- & --- & --- & --- & --- & --- & --- & --- & --- & 1 \\
\hline --- & --- & --- & --- & --- & --- & --- & --- & --- & --- & --- & 1 \\
\hline 74 & 71 & 36 & 75 & 69 & 71 & 72 & 73 & 71 & 71 & 46 & 60 \\
\hline
\end{tabular}

